\title{
LATTICE VECTOR QUANTIZATION FOR IMAGE CODING USING EXPANSION OF CODEBOOK
}

\author{
R. R. Khandelwal ${ }^{1}$, P. K. Purohit ${ }^{2}$ and S. K. Shriwastava ${ }^{3}$ \\ ${ }^{1}$ Shri Ramdeobaba College Of Engineering and Management, Nagpur \\ richareemaerediffmail.com \\ ${ }^{2}$ National Institute of Technical Teachers' Training \& Research, Bhopal \\ purohit_pk2004@yahoo.com \\ ${ }^{3}$ Shri Balaji Institute of Technology \& Management, Betul \\ skshriwastava@gmail.com
}

\begin{abstract}
We present a method for image coding which uses expansion of basic codebook, based on energy of the vectors, for assigning lattice points to the incoming vectors. Two expansions of the basic codebook are used. Since in this method range or radius of the codebooks is based on the energy of the vectors of input image, for different test images we get different basic and expansion codebooks. If the radius of the codebook is very small then mapping of many points may be beyond the range of the codebook, if the radius is very large then time taken to find out the nearest lattice points may be more. So by using this method problem of lattice codebook overload can be bypassed as well as search of vector is reduced because instead of using one small or large codebook, codebook is expanded as and when required. Perceptual performance of peppers, barbara and goldhill images are shown with their MSE and PSNR. Execution time required getting reconstructed image using single codebook and expanded codebook is compared.
\end{abstract}

\section{KEYWORDS}

Lattice Vector Quantization, Basic codebook, Expansion codebook

\section{INTRODUCTION}

Uncompressed Multimedia data requires considerable storage space, transmission bandwidth and computational time. Image compression is required for effective storage and transmission of images. There are two approaches of image compression, lossless and lossy. In lossless compression scheme, the reconstructed image after compression is numerically identical to the original image. In lossy image compression system a transform block is followed by a quantization block. A huge variety of approaches to deal with the task of image coding with lossy compression based on wavelet and vector quantization has been developed. In Vector Quantization same codebook is required by both the encoder and the decoder for quantization. To satisfy this requirement the codebook is also included with the compressed data. As a result, the overall compression ratio may be significantly reduced, especially for large codebooks or high dimensionality of vectors in the case of VQ. Examination of various Vector Quantization techniques shows that a majority of them suffer from high computational cost that is associated with the generation of an appropriate codebook. This implies that they require either a long time to encode the images or complex hardware to make easy the computation in an acceptable period of time. It is possible, however based on the theory of $n$-dimensional lattices to develop a family of quantizers that are free from this drawback. The resulting lattice quantizers offer computational simplicity for image coding because of their regular structure. Lattice quantization does not require transmission of the complete codebook due to its regular structure and as a result, it is

DOI : 10.5121/ijma.2012.4402 
The International Journal of Multimedia \& Its Applications (IJMA) Vol.4, No.4, August 2012

possible to generate the codebook independently at both the encoder and decoder end. Due to simplicity of generating the codebook, lattice quantization delivers a superior speed as compared with other types of quantization. Fast encoding and decoding algorithms for different lattices have been proposed in [1], which makes the computation fast.

In [2] and [3] basic codebook with two expansion codebooks is used. In [3] author used only even value lattice points of $\mathrm{E}_{8}$ lattice with ball type expansion for mobile audio coding. In [4] quantization technique proved efficient for TCX coding of wideband speech signals at $16 \mathrm{KBITS} / \mathrm{S}$, while in [5] voronoi extension for extending fixed rate lattice codebooks in bit rate and support region is used for wideband TCX speech coding with 32KBITS/S. A scaling procedure is required to handle the overload in the largest codebook [4]. A method for encoding long vectors at low bit rate is proposed in [6] for speech and audio coding. In [3] fix energy is used for each codebook i. e. energy of the codebooks remains same for all images, while in this work codebook limits or length of the codebooks decided according to the image. This eliminates codebook overload problem. For implementation $\mathrm{D}_{4}$ lattice is used.

\section{VQ USING LATTICE STRUCTURE}

In vector quantizers (VQ) a group of vectors are mapped to a single vector or codeword. A collection of codewords is called a codebook. In VQ, a group of vectors not having a regular shape while a lattice is a regular arrangement of points in $\mathrm{R}^{\mathrm{n}}$. The codewords of a lattice quantizer are lattice points. The codebook of lattice quantizer is obtained by selecting finite number of lattice points or codewords out of infinite lattice points. So the lattice codebook $\mathrm{C}$ whose bitrate is $\mathrm{R}$ bits/sample is formed by selecting $\mathrm{C}=2^{\mathrm{nR}}$ lattice points about the origin [7].

The codebook radius $\mathrm{m}$ is the normalized distance to the farthest lattice points from the origin. The numbers of lattice points for different codebook radius $\mathrm{m}$ for different lattices and with spherical and pyramidal truncation have been calculated in [8]. If $\mathrm{X}$ is a vector, the codebook radius truncates the vector space into the granular and overload regions such as-

$$
\begin{aligned}
& \text { Granularregion: } \mathrm{CB}^{g r}(m)=\left\{X \in R^{n}:\|X\| \leq m\right\} \cdots \cdots \cdots(1) \\
& \text { Overloadregion: } \mathrm{CB}^{\text {or }}(m)=\left\{X \in R^{n}:\|X\|>m\right\} \cdots \cdots \cdots(2)
\end{aligned}
$$

When some fix radius of codebook is used, it means length of the codebook is fixed. There may be the possibility of overload error in the computation. One solution to that is rescaling the vectors and makes them in the range of codebook. This degrades the quality of reconstructed image. Another solution is expanding the codebook when the vectors are in overload region. Here in this work two expansions of basic codebook according to the energy of the vector are used to overcome overload error.

\section{LVQ WITH EXPANSION OF CODEBOOK}

\subsection{Encoder Architecture}

Figure 1 illustrates the encoder and decoder architecture of the LVQ with expansion of codebook. A wavelet transform is used to decompose the image into a number of levels. We are using $\mathrm{D}_{4}$ lattice, which is optimum lattice in four dimensions, so subbands are reshaped into ZxL size, where $\mathrm{Z}$ depends on the size of input image and wavelet decomposition levels and $\mathrm{L}$ is 4 (dimension of the vector). For example if an image having size 512x512 and it is decomposed upto three level then the subband size will be $64 \times 64$ when it is reshaped to get a vectors each having four values the reshaped matrix will be of size $1024 \times 4$. So the value of $Z$ in this case is 
The International Journal of Multimedia \& Its Applications (IJMA) Vol.4, No.4, August 2012

1024. The lowest frequency subband is coded using Huffman lossless coding. The other three subbands are coded using codebook expansion LVQ. Then indexes are assigned to the coded vectors. From these indexes at the decoder, vectors are generated. By applying inverse wavelet transform on these vectors image is reconstructed at the decoder output.

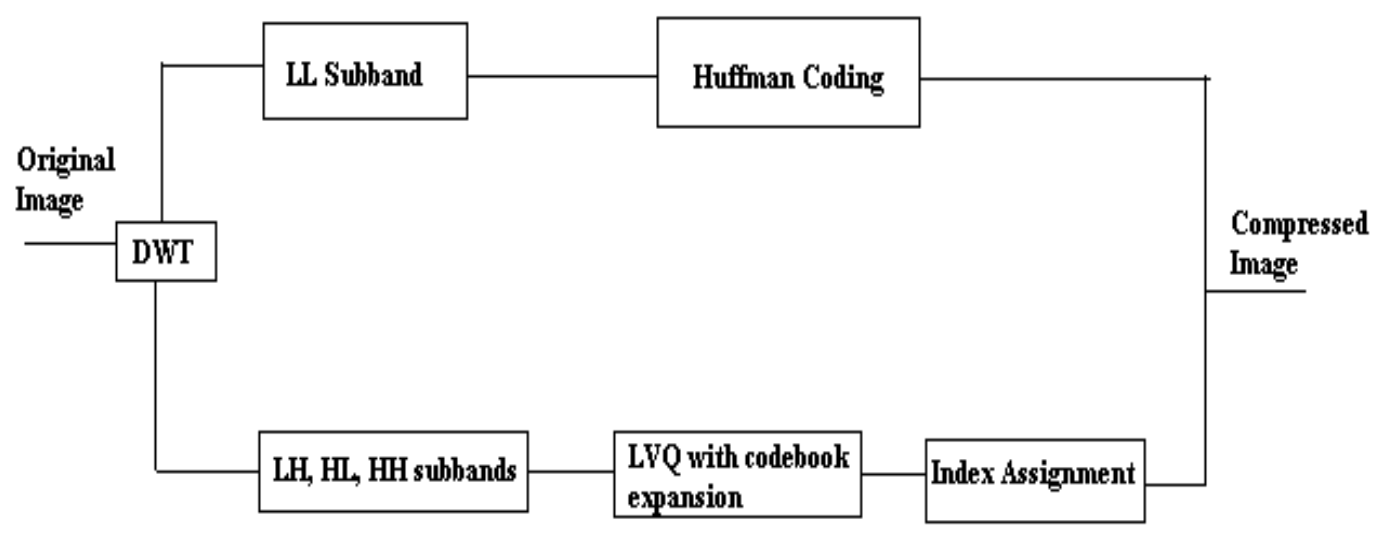

a) Encoder Architecture

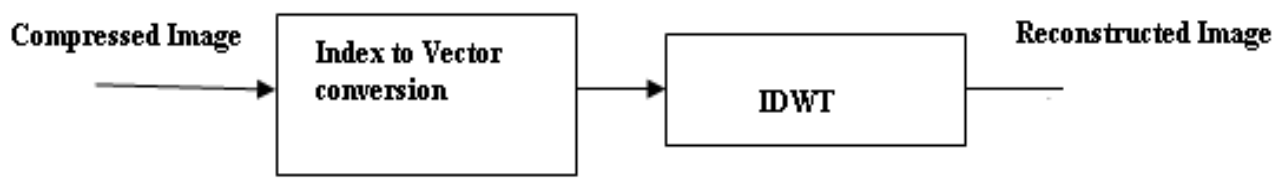

b) Decoder Architecture

Figure 1: a) Encoder Architecture b) Decoder Architecture of an image compression system

\subsection{Design of Expansion Codebook}

This quantization system consists of a basic codebook and two expansion codebooks. In our work energy of the codebooks are not previously decided, it changes according to the image. Minimum value of transformed coefficient and maximum value of transformed coefficient are obtained from the transformed image. From these two values a complete range of the codebook is derived which is from 0 to Upper Limit $(\mathrm{UL})$ where $\mathrm{UL}=\left[4 \mathrm{x}\right.$ (max. of transform coefficient) $\left.{ }^{2}\right]$.This range is further subdivided into three subranges as follows-

Basic codebook- 0-round (UL/3)

First Expansion Codebook- round (UL/3) - 2 x round (UL/3)

Second order Expansion Codebook- 2 x round (UL/3)-UL

Figure 2 shows expansion of codebook based on energy of the vector. Energy of the incoming vector decides whether the closest lattice point is in basic codebook or in first order expansion codebook or in second order expansion codebook. Basic codebook is expanded to first order when the energy of the vector is greater than the maximum limit of the basic codebook and below the upper limit of the first order expansion codebook. If the vector energy not in the range of first 
The International Journal of Multimedia \& Its Applications (IJMA) Vol.4, No.4, August 2012

order expansion codebook it means the nearest neighbor of incoming vector in the lattice lies in the second order expansion codebook.

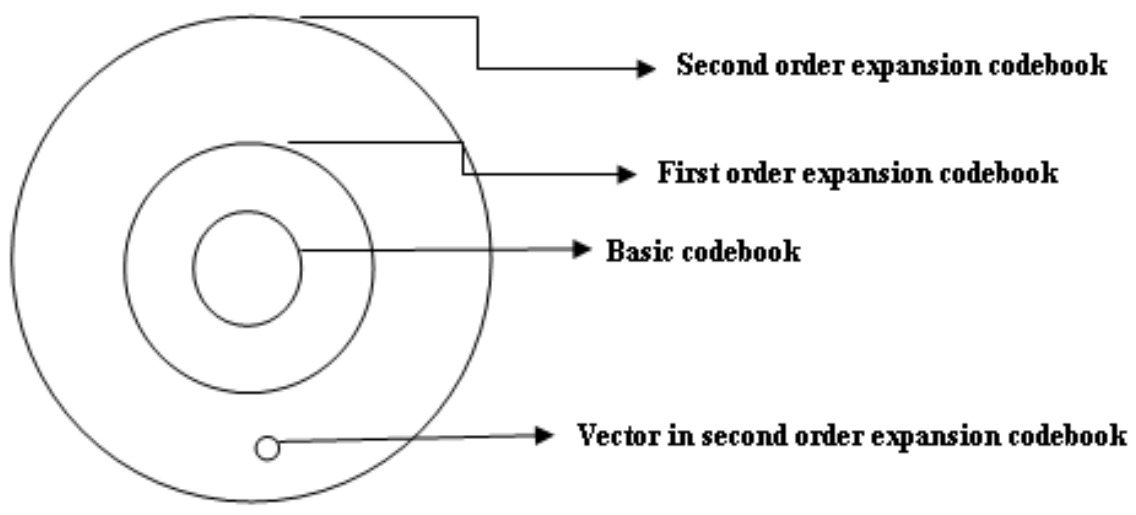

Figure 2: Codebook expansion based on energy

\subsection{Index Allocation}

In a lattice codebook all codevectors are arranged in lexicographic order, accordingly indexes are assigned to those vectors. In this method three codebooks are used, so the index to the codeword contains the codebook type and the index of that vector in corresponding codebook. Number of bits required to represent index is the sum of the bits required to represent that index and bits required to represent type of codebook.

\section{Application to Image Coding}

LVQ with codebook expansion is applied to a wavelet based image coder with wavelet decomposition level-3 using daubechies wavelet. Since the lowest frequency subband is strongly correlated with the original image Huffman coding is applied to this subband.

\subsection{Perceptual performance}

Perceptual performance of single codebook LVQ and expanded codebook LVQ on peppers, barbara and goldhill images of 512x512 are shown in Figure 3. Mean Square Error (MSE) and Peak Signal to Noise Ration (PSNR) are calculated for each image in both cases. It is found that PSNR value for an image is nearly same in both cases. 


\section{Original Images}
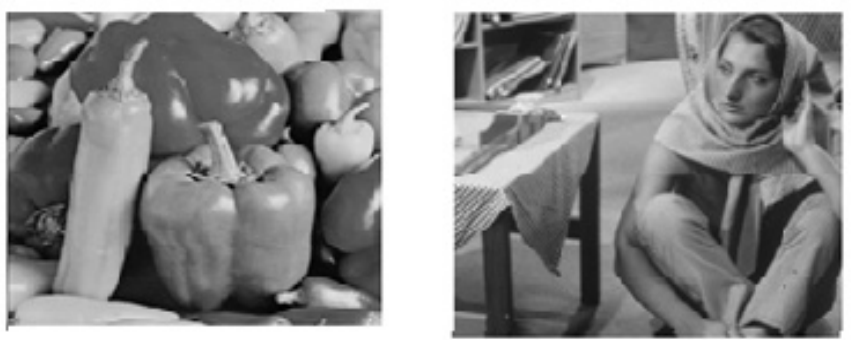

Reconstructed Images using Single Codebook

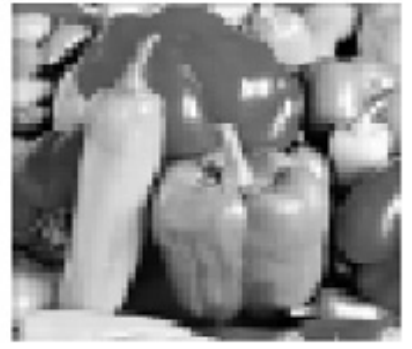

MSE $=336.1266$

PSNR $=22.8658$

Reconstructed Images using Expanded Codebook

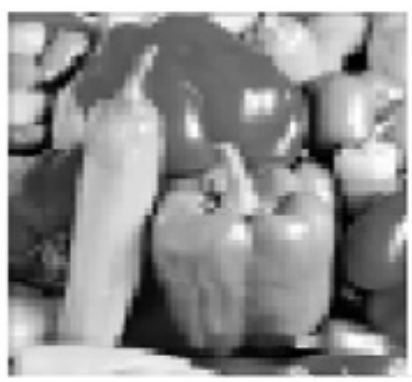

MSE $=337.144$

PSNR $=22.8526$

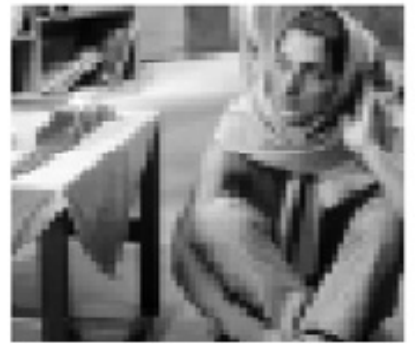

MSE $=497.8535$

PSNR $=21.1598$
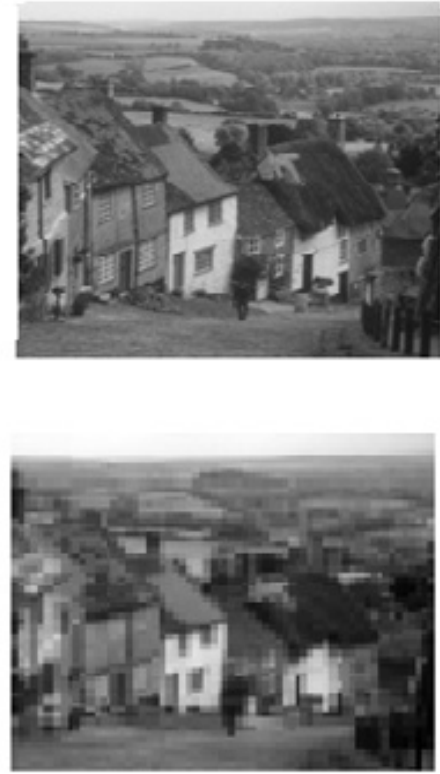

MSE $=259.764$

PSNR $=\mathbf{2 3 . 9 8 5}$

Figure 3: Perceptual performance of Single Codebook LVQ and Expanded Codebook LVQ on peppers, barbara and goldhill images

\subsection{Comparison on time required for execution with single codebook and expanded codebook}

Following table compares the execution time required with single codebook and expansion codebook. In both cases $\mathrm{D}_{4}$ lattice and Pyramidal truncation is used to quantize the vectors of four level wavelet coefficients. Table 1 shows that time required to get reconstructed image with expansion of codebook is less as compared to single codebook. 
The International Journal of Multimedia \& Its Applications (IJMA) Vol.4, No.4, August 2012

Table 1: Comparison of CPU time required to get reconstructed image using single codebook and expansion of codebook

\begin{tabular}{|c|c|c|}
\hline Methods & $\begin{array}{c}\text { CPU time (s) for } \\
\text { peppers }\end{array}$ & CPU time (s) for goldhill \\
\hline Expansion of Codebook & 115.8594 & 131.7656 \\
\hline Single Codebook & 268.8906 & 307.4063 \\
\hline
\end{tabular}

\section{Conclusions}

Lattice Vector Quantization performance depends on the length of the codebook. If number of lattice points is less in a codebook, time required to get quantized lattice point will be less but at the same time quality of reconstructed image degraded. If the size of the codebook is large then the time required to search nearest vector will be more. So to avoid these situations one solution is there, which we have used in this work, expansion of codebook as and when needed. This technique eliminates overload error in the codebook. When compared with single codebook system it requires less time for encoding and decoding.

\section{REFERENCES}

[1] J. H. Conway and N. J. A. Sloane, (1982). Fast Quantizing and Decoding Algorithms for Lattice Quantizers and Codes. IEEE transaction on Information Theory, Vol. IT-28, pp. 227-232.

[2] Bo Hang, Lei Yuan, Zhao Wu, (2009) "A Variable bit Rate Lattice Vector Quantization Method for Audio Coding", in Proc. of International Symposium on Web Information Systems and Applications (WISA'09), Nanchang, P. R. China, pp. 088-091.

[3] Bo Hang, Xiaochen Wang, Ruimin Hu, Ye Ma, Zhao Xie, (2011) "An Even Grid Based Lattice Vector Quantization Algorithm for Mobile Audio Coding”, Journal of Multimedia, Vol. 6, No. 3, pp. 261-268.

[4] M. Xie and J. P. Adoul, "Embedded Algebraic Vector Quantization (EAVQ) with Application to Wideband Audio Coding", in Proc. of IEEE International Conference on Acoustics, Speech and Signal Processing (ICASSP-1996), pp. 240-243.

[5] Stephane Ragot, Bruno Bessette and Roch Lefebvre, "Low-Complexity Multi-Rate Lattice Vector Quantization With Application to Wideband TCX Speech Coding at 32 KBIT/S", in Proc. of IEEE International Conference on Acoustics, Speech and Signal Processing (ICASSP-2004), pp. I-501-I504, 2004

[6] Adriana Vasilache, "Fast Low Bit Rate Lattice Entropy Coding For Speech and Audio Coding", $19^{\text {th }}$ European Signal Processing Conference (EUSIPCO 2011), Barcelona, Spain, pp. 719-723.

[7] Won-Ha Kim, Yu-Hen Hu and Truong Q. Nguyen, "Wavelet - Based Image Coder with Entropy Constrained Lattice Vector Quantizer (ECLVQ)", IEEE transactions on circuits and systems-II: Analog and Digital Signal Processing, 1998, Vol-45, No.8., pp. 1015-1030.

[8] M. Barlaud, P. Sol'e, T. Gaidon, M. Antonini, and P. Mathieu, " Pyramidal Lattice Vector Quantization for Multiscale Image Coding”, IEEE Trans. Image Processing, 1994, Vol. 3, no. 4, pp. 367-381. 